\title{
Contributions of nonneuronal brain cells in substance use disorders
}

\author{
Kathryn J. Reissner ${ }^{1}$ and Mikhail V. Pletnikov ${ }^{2}$ \\ Neuropsychopharmacology (2020) 45:224-225; https://doi.org/10.1038/s41386-019-0494-5
}

\section{INTRODUCTION}

Investigations into the neurobiology of substance use disorders (SUD) have historically focused on neurons. However, recent years have brought a notable shift toward recognition of contributions of nonneuronal cells to SUDs. Here, we provide a brief overview of this emerging topic and suggest future research directions.

\section{DRUG EFFECTS ON GLIAL CELLS}

Accumulating evidence indicates that drug exposure can have dynamic and long-lasting effects on glial cells in the brain, including astrocytes, microglia, and oligodendrocytes. Among the first reported effects of rodent drug self-administration on astrocytes was decreased expression of mediators of glutamate homeostasis in the nucleus accumbens following self-administration of cocaine, heroin, nicotine, and ethanol. Using the membrane-associated Lck-GFP fluorescent reporter, more recent studies indicate that extinction from cocaine self-administration is associated with reduction of structural properties and synaptic co-localization of astrocytes in the nucleus accumbens [1], further suggesting adverse effects of contingent cocaine exposure on neuron-astrocyte communication.

Inflammatory glial responses are also observed following drug use, particularly following chronic opiate treatments associated with tolerance and hyperalgesia. For example, upregulation of proinflammatory cytokines and toll-like receptor signaling on microglia and astrocytes is associated with these processes. However, engagement of these pathways following selfadministration has remained less clear. Notably, recent reports demonstrate that self-administration of cocaine also leads to activation of microglia within the reward circuitry $[2,3]$. Likewise, consequences of drug self-administration on oligodendrocytes are largely unknown. While there have been reports on effects of drug use on myelination and white matter integrity, detailed consequences to structure and activity of oligodendrocytes remain obscure. Intriguingly, a recent transcriptome analysis within the prefrontal cortex of male rats revealed upregulation of markers of oligodendrocyte maturation and differentiation following heroin self-administration [4].

\section{ROLES OF GLIAL CELLS IN BEHAVIORS ASSOCIATED WITH SUD} Beyond the effects of drugs on properties of nonneuronal cells, a growing number of investigations have also assessed the functional contributions of glial cells to behaviors associated with SUDs. For example, ligand stimulation of astrocyte-specific Gq DREADD receptors in the nucleus accumbens leads to reduced reinstatement to cocaine seeking [5]. Further, VTA astrocytes drive avoidance behaviors via stimulation of GABAergic neurons, and stimulation of VTA astrocytes can block cocaine conditioned place preference [6]. Selective overexpression of the oligodendrocyte precursor-specific protein, Sox10, in the prefrontal cortex decreases motivation to self-administer heroin [4]. Future studies should evaluate whether and how oligodendrocyte activity elsewhere in the brain might influence drug taking or relapse. These results collectively suggest that manipulation of glial cells can alter drug-seeking behaviors, and in particular that stimulation of glial cells within the reward circuitry may in some cases oppose drug seeking.

In summary, accumulating evidence suggests that glial cells are critical players in the complex mechanisms of drug seeking, and may represent viable candidates for SUD treatment strategies. In order to identify new glia-based therapeutic targets, continued studies will be required to elucidate more complete glia-specific adaptations associated with SUD, and how amelioration of these adaptations can stem vulnerability to relapse.

\section{FUNDING AND DISCLOSURE}

Funding support provided by DHHS R01DA041455 (KJR) and R01DA041208, P50MH094268, and R01MH083728 (MVP). The authors declare that they have no conflict of interest.

\section{ADDITIONAL INFORMATION}

Publisher's note: Springer Nature remains neutral with regard to jurisdictional claims in published maps and institutional affiliations.

\section{REFERENCES}

1. Testen A, Sepulveda-Orengo MT, Gaines CH, Reissner KJ. Region-specific reductions in morphometric properties and synaptic colocalization of astrocytes following cocaine self-administration and extinction. Front Cell Neurosci. 2018;12:246.

2. Cotto B, Li H, Tuma RF, Ward SJ, Langford D. Cocaine-mediated activation of microglia and microglial MeCP2 and BDNF production. Neurobiol Dis. 2018; 117:28-41.

3. Wang ZJ, Martin JA, Gancarz AM, Adank DN, Sim FJ, Dietz DM. Activin A is increased in the nucleus accumbens following a cocaine binge. Sci Rep. 2017;7:43658.

4. Martin JA, Caccamise A, Werner CT, Viswanathan R, Polanco JJ, Stewart AF, et al. A novel role for oligodendrocyte precursor cells (OPCs) and Sox10 in mediating cellular and behavioral responses to heroin. Neuropsychopharmacology. 2018:43:1385-94.

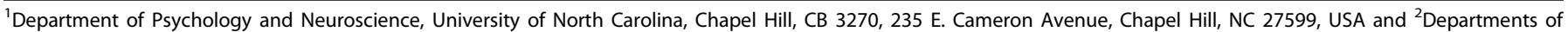
Psychiatry and Behavioral Sciences and Neuroscience, Johns Hopkins University School of Medicine, 600 North Wolfe Street, CMSC 8-117, Baltimore, MD 21287, USA

Correspondence: Kathryn J. Reissner (reissner@unc.edu) or Mikhail V. Pletnikov (mpletni1@jhu.edu)
}

Published online: 2 September 2019 
5. Scofield MD, Boger HA, Smith RJ, Li H, Haydon PG, Kalivas PW. Gq-DREADD selectively initiates glial glutamate release and inhibits cue-induced cocaine seeking. Biol Psychiatry. 2015;78:441-51.
6. Gomez JA, Perkins JM, Beaudoin GM, Cook NB, Quraishi SA, Szoeke EA, et al. Ventral tegmental area astrocytes orchestrate avoidance and approach behavior. Nat Commun. 2019;10:1455.

\title{
TRPV1 and MOR working in tandem: implications for pain
} and opioids use

\author{
Nasser Abdullah ${ }^{1}$ and Christophe Altier ${ }^{1}$ \\ Neuropsychopharmacology (2020) 45:225-226; https://doi.org/10.1038/s41386-019-0516-3
}

Opioids are widely used medications for the relief of moderate to severe pain. While they remain one of the strongest analgesics for pain associated with cancer, trauma, or surgery, prolonged treatment often comes with undesirable side effects, including analgesic tolerance that causes dose escalation and addiction.

Three opioid receptors: mu-, delta-, and kappa-opioid receptors (MOR, DOR, and KOR, respectively) are found in the afferent pain pathway and participate in opioid-induced analgesia [1]. These receptors respond to exogenous (i.e morphine) and endogenous opioids ( $\beta$-endorphin, enkephalins, and the dynorphins) that exert efficient inhibitory control of pain at sites of inflammation and in the central nervous system. The endogenous opioid system represents an evolutionarily important pain-coping strategy during tissue healing and several studies have shown that opioid receptors on afferent nociceptors respond to peripherally acting endogenous opioids released by immune cells, including CD4 ${ }^{+} \mathrm{T}$ cells that are recruited in the later phase of inflammation [2]. Overall, pain sensitization and opioid signaling appear intertwined in the establishment of chronic inflammatory pain. Activation of the mu-opioid receptor (MOR) by opioids results in the binding of $\beta$-arrestin2 to the receptor. This interaction prevents receptor signaling, and elicits desensitization, which in turn reduces the pain-relieving effect and requires increased opioid administration, enhancing the unwanted side effects of MOR activation [3]. Previous work reported an improvement of the opioid therapeutic window in acute inflammation, suggesting a mechanism by which inflammation renders opioid receptors to be more responsive [4]. We recently identified the transient receptor potential vanilloid type 1 (TRPV1), a main target of inflammatory mediators, as a central hub protein that primes pain-relieving effects of opioids. MOR is predominantly expressed in TRPV $1^{+}$ nociceptors and chemical stimulation of the TRPV1 channel was found to prevent $\mathrm{G}$ protein-coupled receptor kinase 5-dependent phosphorylation of agonist-bound MOR [5]. We found that activation of TRPV1 diverts the MOR effector $\beta$-arrestin2 to the nucleus, and this process coincides with enhanced mitogenactivated protein kinases (MAPK) signaling. With $\beta$-arrestin2 removed from the membrane-anchored MOR, the receptor is free of $\beta$-arrestin 2 and hence unable to desensitize and internalize. We next used the complete Freund's adjuvant (CFA) model of chronic inflammatory pain to show that TRPV1 knockout mice do not
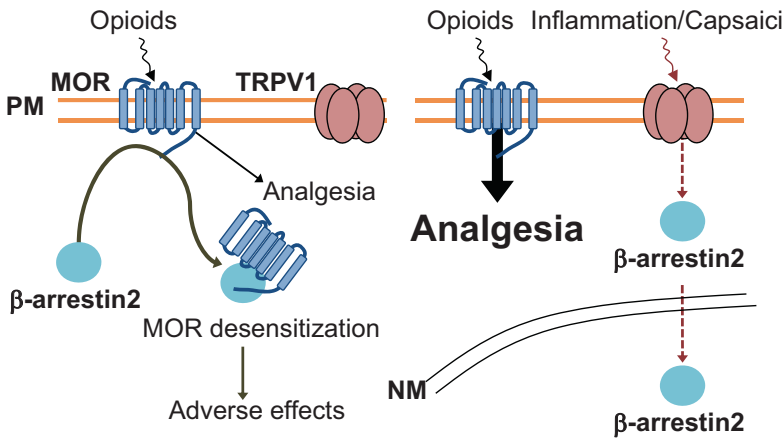

Fig. 1 Illustration of TRPV1-MOR interplay with (right) and without (left) inflammation. MOR desensitization, mediated by $\beta$-arrestin2 recruitment to the receptor, promotes analgesic tolerance. Activation of TRPV1 with capsaicin, or during inflammation, prevents $\beta$ arrestin2-biased signaling of MOR, and thus enhances analgesia

exhibit endogenous opioid analgesia during resolution of inflammation. Finally, using morphine-treated animals, we found that absence of TRPV1 expression promotes peripheral opioid receptor desensitization. Altogether, our findings suggest that agonists of TRPV1 prevent $\beta$ arrestin2-biased signaling of MOR, which enhances analgesia by maintaining peripheral opioid receptor function (Fig. 1) [6]. As chronic inflammatory conditions like arthritis or inflammatory bowel diseases are often associated with persistent pain, further studies will reveal whether the dysregulated interplay between TRPV 1 and $\beta$-arrestin 2 contributes to the transition from acute to chronic pain.

With current research efforts focused on optimizing new types of opioids that circumvent the adverse side effects, these findings could lead to new combination therapies using TRPV1 agonists like vanilloids and cannabinoids as effective analgesics that may also be useful to prevent opioid tolerance.

\section{FUNDING AND DISCLOSURE}

The senior author is a Canada Research Chair in Inflammatory pain. C.A. is supported by the Canadian Institutes of Health Research (ClHR) and the Natural Sciences and Engineering Research Council of Canada (NSERC). The authors declare no competing interests.

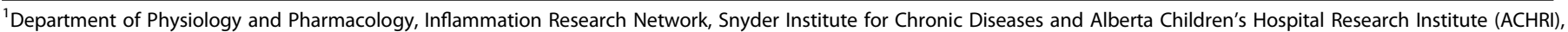
University of Calgary, Calgary, AB T2N 4N1, Canada

Correspondence: Christophe Altier (altier@ucalgary.ca)
}

Published online: 9 September 2019 